\title{
Neuronal MD2 Induces Long-term Mental Impairments in Septic Mice by Facilitating Necroptosis and Apoptosis
}

\section{Zhongmin Fan}

fourth military medical unixersity

Lu Yin

fourth military medical university

Yi Li

fourth military medical university

Hongwei Ma

xijing hospital

You Wu

xijing hospital

Xiaofeng Guo

Fourth Military medical university

Lixia Du

fourth military medical university

Jiajia Wang

xijing hospital

Shiquan Wang

xijing hospital

Wugang Hou

xijing hospital

Hailong Dong

xijing hospital

Zongping Fang

xijing hospital

Xijing Zhang ( $\nabla$ xjzhang0806@163.com )

Fourth Military Medical University: Air Force Medical University https://orcid.org/0000-0001-8719-1142

\section{Research Article}

Keywords: Sepsis-associated encephalopathy, Mental impairments, Neuronal death, Myeloid differentiation factor 2 (MD2), Programmed cell death (PCD) 
Posted Date: October 26th, 2021

DOl: https://doi.org/10.21203/rs.3.rs-995338/v1

License: (c) (i) This work is licensed under a Creative Commons Attribution 4.0 International License. Read Full License 


\section{Abstract}

Sepsis-associated encephalopathy (SAE) is a complication of sepsis that has high morbidity rates. Longlasting depression is a major mental health disorder in patients with SAE that results in a substantial decrease in quality of life and economic burden. However, the underlying mechanism of SAE is unclear, and effective treatments are not available. In the current study, we explored the role of apoptosis and necroptosis related to depression in sepsis. In a mouse model of cecal ligation and puncture (CLP), we detected mental impairments by the open field test, elevated-plus maze and forced swimming test on the fourteenth day. Moreover, apoptosis- and necroptosis-associated proteins and morphological changes were examined in the hippocampus of the septic mice. These mice showed depression-like behaviors at 14 days after CLP, with substantial increases in neuronal apoptosis and necroptosis. Importantly, we found that apoptosis and necroptosis were related according to Ramsay's rule in the brains of the septic mice. Inhibiting the function of MD2, the crosstalk mediator of apoptosis and necroptosis, in neurons effectively reduced neuronal loss and alleviated depression-like behaviors in the septic mice. These results suggest that neuronal death in the hippocampus contributes to the mental impairments in SAE and that inhibiting neuronal MD2 is a new strategy for treatment of mental health issues in sepsis by inhibiting necroptosis and apoptosis.

\section{Introduction}

Sepsis is a life-threatening organ dysfunction caused by a dysregulated host response to infection [1], which is a major cause of death and disability [2]. For septic patients, sepsis-associated encephalopathy (SAE) is a common complication that has a high mortality rate [3]. As a diffuse cerebral dysfunction without direct central nervous system (CNS) infection, SAE results in different levels of consciousness, such as delirium, coma and acute mental dysfunction, in patients [4]. Moreover, $10-58 \%$ of survivors of SAE were found to suffer from long-term depression $[5,6]$. However, the underlying mechanism of longlasting depression in patients with SAE is still unclear.

As a regulated cell death pathway, programmed cell death (PCD), including apoptosis and necroptosis, plays important roles in sepsis. Previous studies have extensively explored the effects of apoptosis in SAE [7-9]. However, whether necroptosis is involved in SAE is unclear. Therefore, elucidating the underlying mechanism of apoptosis and necroptosis in neuronal loss in SAE may lead to treatments for long-term depression in patients with this condition. Our previous study confirmed that myeloid differentiation factor 2 (MD2) is the crosstalk mediator of various PCDs and that inhibition of MD2 effectively reduced stroke injury [10]. Nevertheless, the role of MD2 in the pathology of SAE has not been demonstrated.

In the current study, we proved that neuronal apoptosis and necroptosis are closely related to the longlasting depression of mice with SAE. In addition, we demonstrated for the first time that MD2 is increased in hippocampal neurons. Furthermore, by using CaMKII-Cre; MD2fl/fl mice, we proved that MD2 in excitatory neurons is the key mediator of apoptosis and necroptosis in the hippocampus. Most 
importantly, a blood-brain barrier-penetrating peptide that perturbed the function of MD2 substantially alleviated the depression of the mice with SAE by inhibiting neuronal apoptosis and necroptosis. Our study provides new insights into relieving sepsis-associated depression, suggesting an effective remedy for clinical treatment.

\section{Materials And Methods}

Animals

Male C57/BL6 mice aged 8 weeks (20-25 g) were purchased from Charles River Co., Ltd. (China, Beijing). For generation of conditional MD2 knockout (cKO) mouse lines with deletion of exon 1, male homozygous MD2fl/fl mice on the C57BL/6J genetic background were crossed with female MD2fl/+ mice carrying Cre. Heterozygous MD2fl/+ mice were generated by crossing female MD2 cassette (MD2 cassette/+) mice carrying a targeted MD2 allele on the C57BL/6J genetic background with male protamine-Flp mice. CaMKII-Cre (\#5359) mice with a C57BL/6J genetic background were obtained from The Jackson Laboratory. PCR genotyping of CaMKII cKO mice was performed using the following set of oligonucleotide primers: floxed allele, forward, 5'-TCTCAGTACTTCGGAGGCAGGATGA-3', reverse, 5'TACCCTCCTTTCACTCC CTCGTTCC-3'; Cre allele, forward, 5'-GGTTCTCCGTTTGCACTCAGGA-3', reverse, 5 -CCTGTTGTTCA GCTTGCACCAG- $3^{\prime}$. Mice were housed under standard conditions $\left(22 \pm 2^{\circ} \mathrm{C}\right.$, humidity 50 $\%$, and 12 h light-dark cycle from 8 a.m. to 8 p.m.) and had free access to food and water. All animal procedures were carried out in accordance with the guidelines for the Care and Use of Laboratory Animals of Fourth Military Medical University.

Antibodies and reagents

The following primary antibodies were used: rabbit anti-RIPK1/RIP1 (phospho Ser166) antibody (AGR66476, Arigo), rabbit anti-MLKL (phospho S345) antibody (ab196436, Abcam), rabbit anti-caspase-3 antibody (\#9662, CST), rabbit anti-cleaved caspase-3 (Asp175) antibody (\#9661, CST), rabbit anti- $\beta$-actin (13E5) antibody (\#4970, CST), rabbit anti-MD2 antibody (No. GTX85517, GeneTex), and mouse anti-NeuN antibody [1B7] (ab104224, Abcam). The secondary fluorophore-bound IgGs (Alexa Fluor series) for immunofluorescence (IF) included donkey anti-rabbit IgG H\&L (Alexa Fluor® 594) (ab150064, Abcam) and donkey anti-mouse IgG H\&L (Alexa Fluor ${ }^{\circledR}$ 488) (ab150105, Abcam). Inhibitors including Z-VAD-FMK [ab120487, Abcam, $5 \mu \mathrm{g} / \mu \mathrm{l}$, intracerebroventricular (icv) administration], necrostatin-1 (Selleck, $3 \mu \mathrm{g} / \mu \mathrm{l}$, icv), protease inhibitor cocktail (No. 16B140042, Biotool) and phosphatase inhibitor cocktail (No. B15002, Biotool) were used. Tat-fused peptides were custom-synthesized by the Peptide Synthesis and Purification Core Facility at GL Biochem. NeuroTrace ${ }^{T M}$ Nissl 500/525 green fluorescent stains were purchased from Invitrogen.

Cecal ligation and puncture (CLP)

The mice were fasted for $12 \mathrm{~h}$ but had free access to water before the surgical procedure. Then, anesthesia was induced by inhalation of $2 \%$ isoflurane through a face mask, and anesthesia was 
maintained with $1.5 \%$ isoflurane. Under sterile conditions, the cecum was ligated with 4-0 silk at the midpoint and punctured once with a 22-gauge needle. Then, the cecum was gently squeezed until a single droplet of fecal material came out of the puncture site. Next, the abdominal cavity was closed in two layers, followed by fluid resuscitation (preheated saline, $20 \mathrm{ml} / \mathrm{kg}$ ), and the animal was returned to its cage. In the sham group, except for ligation and perforation, the rest of the steps were the same as those in the CLP model group.

Peptide design

According to the high affinity between the cold-inducible RNA binding protein CIRP and MD2 [11], we synthesized a blocking peptide to mimic the 106-125 domain of CIRP and link the trans-trans-activating (Tat) transmembrane functional domain (YGRKKRRQRRR) to form Tat-CIRP (YGRKKRRQRRRGRGFSRGGGD RGYGG), which promotes penetration of the BBB. For other details, see our previous study [10].

Western blot analysis

Western blot analysis was carried out on the protein samples from the hippocampus. The mice were sacrificed, and the hippocampus was collected and stored at $-80{ }^{\circ} \mathrm{C}$. Fresh hippocampal tissues were weighed and homogenized in lysis buffer containing protease and phosphatase inhibitors. After lysis on ice for $20 \mathrm{~min}$, the homogenates were centrifuged at $12000 \mathrm{rpm}$ for $10 \mathrm{~min}$ at $4{ }^{\circ} \mathrm{C}$. Then, the supernatant was collected, and $10 \mu \mathrm{l}$ was used for BCA protein quantification. The residual supernatant was denatured with $4 \times$ loading buffer at $100{ }^{\circ} \mathrm{C}$ for 10 min and stored in a $-20^{\circ} \mathrm{C}$ freezer for later use. Subsequently, $10 \mu \mathrm{l}$ of the prepared sample was separated by SDS-PAGE and transferred to a PVDF membrane under a voltage of $100 \mathrm{~V}$ for $90 \mathrm{~min}$. After the membrane was blocked with $50 \mathrm{~g} / \mathrm{l} \mathrm{BSA}$ or $5 \%$ nonfat milk for $2 \mathrm{~h}$, it was incubated with primary antibody overnight in a refrigerator at $4{ }^{\circ} \mathrm{C}$ followed by incubation with HRP-labeled IgG for $2 \mathrm{~h}$ at room temperature. After thorough washing with TBST, the BioRad gel imaging system was used for chemical development.

Nissl staining

For Nissl staining, after deparaffinization and rehydration, the slices were immersed in $100 \%$ ethanol for approximately $5 \mathrm{~min}, 95 \%$ ethanol for $30 \mathrm{~s}$, and $70 \%$ ethanol for $30 \mathrm{~s}$. The samples were then washed in PBS three times, immersed in $1 \%$ Nissl for 8 min, and washed with PBS three times. Next, the samples were immersed in $70 \%$ ethanol for $2 \mathrm{~min}, 80 \%$ ethanol for $2 \mathrm{~min}, 95 \%$ ethanol for $2 \mathrm{~min}, 100 \%$ ethanol for $2 \mathrm{~min}$ (twice) and xylene for $2 \mathrm{~min}$ (twice). Finally, the slices were sealed by neutral gum. For TeuroTraceTM fluorescent Nissl staining, cryosections $(10 \mu \mathrm{m})$ were prepared with standard protocols. Then, the samples were rehydrated in PBS for $40 \mathrm{~min}$ and washed twice for $10 \mathrm{~min}$ in PBS plus $0.1 \%$ Triton X-100. Next, the sections were covered with NeuroTrace stain (1:2000) for at least $20 \mathrm{~min}$. After thorough washing, DAPI-containing mounting tablets were used for mounting.

IF staining 
Mice were deeply anesthetized with $2 \%$ pentobarbital sodium [50 mg/kg, ip) and was rapidly perfused 50 $\mathrm{ml}$ of pre-cooled saline through the heart, followed by slow perfusion with $50 \mathrm{ml}$ of $4 \%$ paraformaldehyde. The brain was taken out and fixed for 2 hours in $4 \%$ paraformaldehyde. Then they were dehydrated with $20 \%$ and $30 \%$ sucrose solutions in sequence until the brain sank to the bottom. And they were made into frozen tissue section in $10 \mu \mathrm{m}$. The brain slices were washed 3 times with PBS for 10 minutes each time. After washing, the slices were blocked with donkey serum and $0.05 \%$ Triton X100 for 2 hours at room temperature, and were incubated with primary antibodies overnight at $4{ }^{\circ} \mathrm{C}$. After washing thoroughly, secondary antibody was add to the slices in a dark box for 2 hours. After thorough washing, DAPI-containing mounting tablets were used to mount the slices. Then, they were placed in a confocal microscope.

Stereotactic injection

Tat-CIRP or inhibitors were injected into lateral ventricles through stereotactic technology. Mice were anesthetized with $2 \%$ pentobarbital sodium ( $20 \mathrm{mg} / \mathrm{kg}$, ip) and fixed in a stereotaxic device. Then, their eyes were covered by erythromycin ointment to prevent injury caused by long-term exposure. After a series of procedures, we identified the coordinates of the lateral ventricles $(A P=-0.5 \mathrm{~mm}, M L=+1.0 \mathrm{~mm}$, $\mathrm{DV}=-1.4 \mathrm{~mm}$ ) and injected relevant drugs at a total volume of $1 \mu \mathrm{l}$.

Open field test (OFT)

The OFT is one of the most commonly used tests to assess anxiety-like behaviors in rodents and allows animals to freely explore open areas surrounded by walls. Under normal conditions, the animals show thigmotaxis (tend to avoid the central region and stay at the peripheral region), and increased or decreased center activity is associated with anxiolysis or anxiogenesis [12]. The mice were placed in an apparatus consisting of a $40 \times 40 \times 40 \mathrm{~cm}$ polyvinyl chloride (PVC) box, and a camera was used to monitor their movements. The tests were all carried out between 6:30 pm and 10:30 pm. After each test, the mice were returned to the cage, and the bottom and sidewalls of the box were cleaned with $75 \%$ alcohol. The next step did not start until the box was dry and there was no smell.

Elevated plus maze (EPM)

An EPM is one of the traditional tests to evaluate anxiety-like behavior. In this test, four elevated arms radiate from a central platform in which two opposing arms are walled and the other two opposing arms are opened. Mice were placed on the central area and allowed to freely explore the maze for a total of 5 min. Mice tended to avoid open or elevated places, which was counterbalanced by their innate curiosity to explore areas that are new to them. More time in the open arms was associated with less anxiety, and more time in the closed arms was related to anxiety. Additionally, the tests were all carried out between 6:30 pm and 10:30 pm. After each test, the mice were returned to the cage, and the arms were cleaned with $75 \%$ alcohol. The next step did not start until the device was dry and there was no smell.

Forced swimming test (FST) 
During the test, mice were subjected to an 8-min swim session including $2 \mathrm{~min}$ in the adaptation stage and $5 \mathrm{~min}$ in the test stage in clear plexiglass cylinders $(30 \mathrm{~cm}$ height $\times 20 \mathrm{~cm}$ diameter) filled with $17 \mathrm{~cm}$ of water $\left(23 \pm 2^{\circ} \mathrm{C}\right)$. The test was performed between $6: 30 \mathrm{pm}$ and 10:30 pm under normal light conditions. The tests were recorded by a digital video camera. The immobility time, which was defined as passive floating with no additional activity other than that necessary to keep the animal's head above the water, was scored by a trained observer. After each test, the mice were placed in an incubator until they recovered.

\section{Statistical analysis}

GraphPad Prism 8.0.2 was used to conduct the statistical analysis. All values except for the murine sepsis score (MSS) and the weights are presented as the mean \pm SEM and were analyzed by using twotailed Student's t test (comparisons of two groups) or one-way analysis of variance (ANOVA) followed by Dunnett's or Tukey's post hoc test (comparisons of more than two groups). Survival curves and comparisons among curves were assessed through the Mantel-Cox log-rank test. A P value less than 0.05 was defined as significant.

\section{Results}

\section{Sepsis induced long-term mental impairments in mice that underwent CLP}

First, we evaluated the severity of sepsis (MSS) according to previous research [13]. The MSS significantly increased during the first 3 days and peaked on the third day. Then, the MSS gradually decreased in the following days (Supplementary Fig. 1A), indicating alleviation of sepsis. Similarly, the weights of the CLP-induced mice declined after the surgery and reached a valley on the third day (Supplementary Fig. 1B). Total distance (TD) as an indicator of OFT is often used to evaluate the general health of animals. Our results showed no difference in TD between the sham-treated and CLP-induced mice at 14 days after sepsis (Supplementary Fig. 1D), which indicated that the evaluation of anxiety and depressive behaviors was reasonable. Then, we analyzed the relationship between MSS and outcome. Through the two-sample T test, we found a significant relationship between the third day score and final mortality (Supplementary Table 1). Then, we established a group trajectory development model according to the MSS and final outcomes. We found the animals can be divided into three groups. The first group showed a low probability of death with MSS $<5$, and the third group presented high mortality with MSS $>10$. The second group showed a balance between survival and death, and MSS $\geq 5$ was the inflection point (Supplementary Fig. 1C; Supplementary Table 2). Therefore, we chose mice with MSS $\geq 5$ on the third day after CLP for subsequent experiments.

Next, we performed a battery of behavioral tests at 14 days after CLP to determine whether the septic mice present depression-like behaviors (Fig. 1A). The OFT (Fig. 1B), EPM (Fig. 1E) and FST (Fig. 1H) were performed. In the OFT, the number of entries to the center zone (Fig. 1C) and the time spent in the center 
zone (Fig. 1D) were decreased in the CLP group compared with the sham group. Similarly, the percentage of time spent in the open arm of the septic mice was significantly reduced in the EPM (Fig. 1G). However, the animals presented no difference in the percentage of entry in the open arm (Fig. 1F). The immobility time of the septic mice was increased in the FST (Fig. 1I). The above results indicated that CLP induced long-term mental impairments in mice.

Then, we explored the possible mechanism of sustaining depression-like behaviors induced by sepsis. Pathological staining showed substantial neuronal death in the hippocampus of the septic mice (Fig. 1J, $\mathrm{K}$ ). These findings indicate the probable mechanism of hippocampal neuronal death underlying the longlasting depression-like behaviors of the septic mice.

\section{Both apoptosis and necroptosis in hippocampal neurons were increased in the septic mice}

Apoptosis and necroptosis are two major PCDs that participate in the pathological processes of sepsis. To investigate their roles in SAE in septic mice, we assessed apoptosis-associated proteins and necroptosis-associated proteins in the hippocampus. Our Western blot results indicated a marked increase in the amounts of p-Ripk1, p-MLKL (necroptosis markers) and cleaved caspase-3 (apoptosis markers) in the hippocampus during the first three days and a peak at $24 \mathrm{~h}$ after CLP surgery (Fig. A, B, C, D; Supplementary Fig. A, B, C, D). Moreover, the IF results demonstrated increased apoptosis and necroptosis in hippocampal neurons at $24 \mathrm{~h}$ after CLP (Fig. 2E, F, G, H; Supplementary Fig. E, F, G, H). These results revealed that apoptosis and necroptosis were increased in hippocampal neurons, which may mediate the pathological process of sepsis.

\section{Apoptosis and necroptosis were related according to Ramsay's rule in the brains of the septic mice}

Next, we tested whether suppression of apoptosis or necroptosis in the hippocampus can alleviate the mental dysfunction induced by sepsis. Necrostatin-1 (Nec-1) (a specific inhibitor of necroptosis [14]) and benzyloxycarbonyl-Val-Ala-Asp-fluoromethyl ketone (Z-VAD-FMK) (a specific inhibitor of apoptosis [15]) were administered intracerebroventricularly to mice immediately after CLP surgery. Then, we observed their behaviors at 14 days after CLP (Supplementary Fig. 3A, B). Surprisingly, we found no differences among the distinct groups in the survival rate and mental disorder-associated behaviors (Supplementary Fig. 3C, D, E, F, G, H). Moreover, there was no significant improvement in the MSS, weight or neuronal loss under inhibitor treatment (Supplementary Figs. 4, 5). These results indicated that inhibiting apoptosis or necroptosis alone could not rescue the depression of the septic mice.

Then, we explored the reasons why inhibition of apoptosis or necroptosis failed to alleviate depression in the septic mice. Our results showed that Nec-1 treatment facilitates the expression of cleaved caspase-3 and that the administration of Z-VAD-FMK accelerates the phosphorylation of RIPK1 (Fig. 3A, B, C, D), which indicated a relationship according to Ramsay's rule between apoptosis and necroptosis. However, synergy using Nec-1 and Z-VAD-FMK increased the death of the septic mice (Supplementary Fig. 3C), which may be attributed to the superimposed toxicity of inhibitors and solvent. 
MD2 synchronously mediated apoptosis and necroptosis, and excitatory neuronal MD2 deletion relieved depression-like behaviors in the septic mice

Given the current unsatisfactory treatments, we attempted to identify a target that affects both apoptosis and necroptosis. MD2 is an essential cofactor protein of TLR4 that participates in the inflammatory response and is a key mediator of apoptosis and necroptosis in stroke models [10]. Here, we explored the expression of MD2 in neurons in the hippocampus of the septic mice. We found a prominent increase in MD2 expression at $24 \mathrm{~h}$ after CLP surgery (Fig. 4A, B, C, D). To further confirm the crucial role of MD2 in the pathology of SAE, we constructed transgenic mice that with specific MD2 knockout in excitatory neurons (see our previous research for details [10]). The expression of apoptosis- and necroptosisassociated proteins was reduced in the hippocampus of the CaMKII-MD2fl/fl mice compared with their littermates (Fig. 5A, B, C, D). Moreover, the amount of HMGB1, a typical damage-associated molecular pattern (DAMP) molecule, was decreased (Fig. 5E, F). Then, we tested proinflammatory factors in the hippocampus of the CaMKII-MD2fl/fl mice. The expression of IL-6 was decreased in the CaMKII-MD2fl/fl mice (Fig. $6 \mathrm{H}, \mathrm{I}, \mathrm{J}$ ). Accordingly, less cell death was observed in the hippocampus of the CaMKII-MD2fl/fl mice than the control mice (Fig. $5 \mathrm{G}, \mathrm{H}$ ).

Then, we performed a series of experiments (Supplementary Fig. 6A) and found that the CaMKII-MD2fl/fl mice exhibited fewer mental impairments 14 days after CLP (Fig. 6A, B, C, D, E, F, G). Moreover, the MSS and weight loss of the CaMKII-MD2fl/fl mice with sepsis were mitigated compared with those of their littermates (Supplementary Fig. 6C, D)

\section{TAT-CIRP injection in the brain alleviated depression-like behaviors and neuronal death in the septic mice}

After demonstrating the critical role of MD2 in SAE, we tried to find a potential clinical treatment for SAE. In our previous study, we designed a short peptide to disturb the function of MD2 [10]. To confirm its utility in the sepsis model, we administered TC via icv injection into the mice during the first 3 days after CLP, and the behaviors were tested on the fourteenth day after CLP (Fig. 7A, B). The TC administration group showed fewer mental symptoms than the CLP group (Fig. 7D, E, F, G, H, I, J). The MSS of the TC group was lower than that of the CLP group without any other treatment (Supplementary Fig. 7A). The weight of the TC group declined quickly during the period of drug delivery; however, the speed and degree of weight recovery were better than those of the saline groups (Supplementary Fig. 7B). There was only a trend of improved survival in the TC-treated group, which was treated with icv administration (Fig. 7C). Neuronal loss in the septic mice was alleviated with TC (Fig. 9A, B). These results show that TC has good application prospects in sepsis.

\section{Discussion}

It has been reported that more than 19 million individuals develop sepsis each year [16]. As one of the major manifestations of SAE, long-term mental dysfunction affects $29 \%$ of surviving septic patients [16, 17], which leads to poor quality of life and a heavy economic burden. However, the concrete mechanism of long-lasting mental impairments in septic patients is not clear. As a result, no effective therapies are 
available. In our current study, we found that the septic mice presented long-term anxiety and depression induced by CLP. Furthermore, increased apoptosis and necroptosis in the hippocampus were detected in these mice, resulting in substantial neuronal loss. We demonstrated that apoptosis and necroptosis were related according to Ramsay's rule, and inhibiting only apoptosis or necroptosis failed to rescue the cognitive symptoms in the septic mice. Importantly, we confirmed that MD2 regulates both apoptosis and necroptosis, and a peptide targeting MD2 reduced both apoptosis and necroptosis while significantly relieving anxiety and depression-like behaviors in the septic mice.

Major depressive disorder (MDD) is common, affecting approximately $16 \%$ of the population worldwide, and is associated with a high disability rate and socioeconomic consequences [18]. The incidence of long-term depression in septic patients is even higher than that in the general population, but little is known about the underlying mechanism. Evidence from imaging, postmortem and other studies suggests that abnormal corticolimbic structures, including the prefrontal cortex, anterior cingulate cortex, amygdala and hippocampus, are responsible for MDD [19]. Significant neuronal loss in the hippocampus of individuals with MDD has been confirmed [20]. Moreover, the expression of apoptosis-associated genes was increased in the frontal cortex of MDD patients. This evidence indicates a close link between corticolimbic neuronal death and depression. However, it is still unclear whether neuronal death is the cause or result of depression. Notably, neuronal loss has already been documented in patients with sepsis. Changes in brain volume, especially atrophy of the hippocampus and cortex, have been reported in sepsis with abnormal neurological symptoms [21]. Some studies have reported that inhibition of the cell death pathway can alleviate long-term cognitive impairments and depression-like behaviors in septic mice $[22,23]$. In the current study, we found a substantial increase in apoptosis- and necroptosisassociated proteins in the first three days after CLP. Accordingly, we also found notable neuronal death in the hippocampus of the mice with sepsis. On the fourteenth day after CLP, the fundamental state was similar to that of the sham-treated mice, indicating that sepsis had been alleviated. However, most of the septic mice showed severe depression-like behaviors. This finding indicated that irreversible severe neuronal death in the hippocampus caused by sepsis may be a main cause of the depression-like behaviors.

Multiple PCDs contribute to the loss of hippocampal neurons. Apoptosis and necroptosis are the main types of PCD that are involved in the pathology of anxiety and depression [24]. Multiple studies have confirmed the increased apoptosis in the hippocampus of mice with SAE [25], which is consistent with our results. And many drugs target on the decrease of apoptosis show significant brain-protective effects $[9,25]$. However, our study showed no benefits of solely inhibiting apoptosis with Z-VAD-FMK or necroptosis with Nec-1. In fact, these drugs not only act on apoptosis but also mediate other mechanisms, such as autophagy, oxidative stress and neuroinflammation, and thus do not directly prove the protective effects of apoptotic inhibition. No studies have been conducted on necroptosis in SAE. However, some studies have focused on the role of necroptosis in the peripheral organs of septic mice. After Nec-1 treatment, the serum levels of proinflammatory factors such as IL- $6, I L-1 \beta$ and TNF- $a$ were decreased [26]. However, some studies have shown that Nec-1 accelerates death in a rat model of CLP and substantially increases the expression of cleaved caspase-3 in hepatocytes [27]. Therefore, the role 
of necroptosis in sepsis and SAE remains unclear. Currently, increasing evidence has shown the mutual transformation between apoptosis and the necroptosis signaling pathway. Our data showed that both apoptosis and necroptosis participated in the pathological mechanisms of SAE. Interestingly, apoptosis and necroptosis are related through Ramsay's rule. This phenomenon indicates that inhibition of just one pathway cannot effectively rescue depression. Notably, in the current study, simultaneous treatment with inhibitors of apoptosis and necroptosis caused more death in the septic mice, which may result from the superposition of toxic effects that exceed their protective effects.

Alternatively, we searched for upstream pathways that can simultaneously regulate apoptosis and necroptosis. Our previous study confirmed that MD2, which acts as an accessory protein of TLR4, can also regulate various cell death pathways [10]. We constructed CaMKII-MD2fl/fl mice with specific knockout of MD2 in excitatory neurons. In the present study, we found significant improvements in the depression-like behaviors of the CaMKII-MD2fl/fl mice with sepsis. However, the MD2-TLR4 complex has been widely explored in sepsis. MD2 was responsible for the recognition and bonding of the membrane component of gram-negative bacteria (lipopolysaccharide) and triggering the TLR4-mediated inflammatory response. Inhibitors of MD2 could reduce inflammation and alleviate tissue injury in sepsis [28]. As the effects of neuroinflammation on MDD have been potently confirmed [29], we could not ignore the effect of MD2 blockade on inflammation in the hippocampus of mice with sepsis. Some systematic reviews and meta-analyses have shown that only IL-6 expression is significantly higher in depression [30]. In accordance with these results, our findings demonstrated that HMGB1 and IL-6 levels were significantly decreased in the CaMKII-MD2fl/fl mice compared with the WT mice at $24 \mathrm{~h}$ after CLP.

The molecular mechanism by which MD2 regulates apoptosis and necroptosis remains unknown and needs further exploration. Our previous study found that MD2 mediated apoptosis and necroptosis by combining with Src-associated substrate in mitosis of $68 \mathrm{KD}$ (Sam68). TAT-CIRP restrained the function of MD2 and reduced brain injury-induced stroke [10]. In this study, we used TAT-CIRP at a dose of 25 $\mathrm{mg} / \mathrm{kg}$ in the brain to reduce neuronal death and effectively relieve SAE symptoms. Sam68, also known as KHDRBS1, participates in the cell cycle, apoptosis and signaling. Sam68 was confirmed to be recruited to the TNF receptor and promote the recruitment and ubiquitylation of RIP [31]. Moreover, Sam68 is a part of the TNF-induced cytoplasmic caspase-8-FADD complex, and its cleavage by activated caspase, especially caspase-8, triggers the apoptosis pathway [32]. However, the mechanism by which MD2 acts on Sam68 and whether other molecules can interact with MD2 remain unclear. More studies on the regulatory mechanisms of MD2 will contribute to its use in the clinic for sepsis.

There are some limitations in this study. First, the CaMKII-MD2fl/fl mice had MD2 knocked out in the neurons of the whole brain and TC was administered via icv injection. Therefore, we could not eliminate the influence of other structures, such as the cortex and amygdala, which are associated with depression. Further studies exploring MD2-mediated neuronal death in the cortex and amygdala are needed. Next, we confirmed the relationships among neuronal death, neuroinflammation and depression in septic mice. Whether neuroinflammation is the cause or the result of cell death is still unknown and needs further investigation. Finally, MD2 is expressed not only in neurons but also in microglia and astrocytes in the 
brain. MD2 in peripheral immune cells can also enter the brain through the damaged brain-blood barrier in septic mice. Thus, we could not ignore the effects of extraneous MD2 on neurons. These issues related to MD2 in sepsis need further exploration.

\section{Conclusions}

In summary, the present study demonstrated for the first time that neuronal apoptosis and necroptosis in the hippocampus contribute to depression-like behaviors in septic mice. We demonstrated that MD2 was the crosstalk mediator of apoptosis, necroptosis and neuroinflammation in the pathology of depression induced by sepsis. Inhibition of MD2 in neurons can reduce neuronal loss and alleviate depression-like behaviors in septic mice. Methods targeting MD2 in neurons may provide new treatment strategies for SAE.

\section{Declarations}

Author contributions ZMF, LY and YL initiated, designed, performed and analyzed the experiments and wrote the manuscript. HWM and YW established the mouse CLP model. SQW and JJW performed transmission electron microscopy. WGH, XFG and LXD performed histology. XJZ, ZPF and HLD conceived the study, supervised the project, designed experiments, and wrote the manuscript.

Funding This work was supported by the National Natural Science Foundation of China (grant number 81871603) for Prof Zhang, the National Natural Science Foundation of China Youth Fund (grant number 81801308) for Prof Fang, and the Natural Science Foundation of Shaanxi Province of China (grant number 2020JQ-446) for Prof Fang.

Data availability The datasets generated and analyzed during the current study are available from the corresponding author on reasonable request.

\section{Compliance with Ethical Standards}

All animal procedures were approved by the Ethics Committee of Fourth Military Medical University in full accordance with the ethical guidelines of the National Institutes of Health for the care and use of laboratory animals.

Conflict of interest The authors declare that they have no competing interests.

\section{References}

1. Singer M, Deutschman CS, Seymour CW, Shankar-Hari M, Annane D, Bauer M, et al. The third international consensus definitions for sepsis and septic shock (sepsis-3). JAMA. 2016;315:801-810. https://doi.org/10.1001/jama.2016.0287. 
2. Napolitano LM. Sepsis 2018: Definitions and guideline changes. Surg Infect (Larchmt). 2018;19:117-125. https://doi.org/10.1089/sur.2017.278.

3. Saito M, Fujinami Y, Ono Y, Ohyama S, Fujioka K, Yamashita K, et al. Infiltrated regulatory t cells and th2 cells in the brain contribute to attenuation of sepsis-associated encephalopathy and alleviation of mental impairments in mice with polymicrobial sepsis. Brain Behav Immun. 2021;92:25-38. https://doi.org/10.1016/j.bb i.2020.11.010.

4. Gofton TE, Young GB. Sepsis-associated encephalopathy. Nat Rev Neurol. 2012;8:557-566. https://doi.org/10.1038/nrneurol.2012.183.

5. Calsavara AC, Rodrigues DH, Miranda AS, Costa PA, Lima CX, Vilela MC, et al. Late anxiety-like behavior and neuroinflammation in mice subjected to sublethal polymicrobial sepsis. Neurotox Res. 2013;24:103-108. https://doi.org/10.1007/s12640-012-9364-1.

6. Streck EL, Comim CM, Barichello T, Quevedo J. The septic brain. Neurochem Res. 2008; 33:21712177. https://doi.org/10.1007/s11064-008-9671-3.

7. Ghrelin prevents neuronal apoptosis and cognitive impairments in sepsis-associated encephalopathy: Retraction. Neuroreport. 2012;23:405. https://doi.org/10.1097/WNR.0b013e328 3515 ce4.

8. Zhang L, Zhang X, Wu T, Pan X, Wang Z. Isoflurane reduces septic neuron injury by ho1mediated abatement of inflammation and apoptosis. Mol Med Rep. 2021;23:155. https://doi.org/ 10.3892/mmr.2020.11794.

9. Bedirli N, Bagriacik EU, Yilmaz G, Ozkose Z, Kavutcu M, Cavunt Bayraktar A, et al. Sevoflurane exerts brain-protective effects against sepsis-associated encephalopathy and memory impairment through caspase $3 / 9$ and bax/bcl signaling pathway in a rat model of sepsis. J Int Med Res. 2018;46:2828-2842. https://doi.org/10.11 77/0300060518773265.

10. Fang Z, Wu D, Deng J, Yang Q, Zhang X, Chen J, et al. An md2-perturbing peptide has therapeutic effects in rodent and rhesus monkey models of stroke. Sci Transl Med. 2021;13:eabb6716. https://doi.org/10.1126/scitranslmed.abb6716.

11. Qiang X, Yang WL, Wu R, Zhou M, Jacob A, Dong W, et al. Cold-inducible rna-binding protein (cirp) triggers inflammatory responses in hemorrhagic shock and sepsis. Nat Med. 2013;19:1489-1495. https://doi.org/10.1038/nm.3368.

12. Kuniishi H, Ichisaka S, Yamamoto M, Ikubo N, Matsuda S, Futora E, et al. Early deprivation increases high-leaning behavior, a novel anxiety-like behavior, in the open field test in rats. Neurosci Res. 2017;123:27-35. https://doi.org/10.1016/j.neures. 2017.04.012. 
13. Shrum B, Anantha RV, Xu SX, Donnelly M, Haeryfar SM, McCormick JK, et al. A robust scoring system to evaluate sepsis severity in an animal model. BMC Res Notes. 2014;7:233. https://doi.org/10.1186/1756-0500-7-233.

14. Cao L, Mu W. Necrostatin-1 and necroptosis inhibition: Pathophysiology and therapeutic implications. Pharmacol Res. 2021;163:105297. https://doi.org/10.1016/ j.phrs.2020.105297.

15. Chang H, Sun F, Tian K, Wang W, Zhou K, Zha D, et al. Caspase inhibitor Z-vad-fmk increases the survival of hair cells after actinomycin-d-induced damage in vitro. Neurosci Lett. 2020;732:135089. https://doi.org/10.1016/j.neulet.2020.135089.

16. Prescott HC, Angus DC. Enhancing recovery from sepsis: A review. JAMA. 2018;319:62-75. https://doi.org/10.1001/jama.2017.17687.

17. Calsavara AJ, Costa PA, Nobre V, Teixeira AL. Prevalence and risk factors for post-traumatic stress, anxiety, and depression in sepsis survivors after icu discharge. Braz J Psychiatry. 2020;43:269276. https://doi.org/10.1590/1516-4446-2020-0986.

18. Zanos P, Moaddel R, Morris PJ, Georgiou P, Fischell J, Elmer GI, et al. NMDAR inhibitionindependent antidepressant actions of ketamine metabolites. Nature. 2016;533:481-486. https://doi.org/10.1038/nature17998.

19. Drevets WC, Price JL, Furey ML. Brain structural and functional abnormalities in mood disorders: Implications for neurocircuitry models of depression. Brain Struct Funct. 2008;213:93-118. https://doi.org/10.1007/s00429-008-0189-x.

20. Lee AL, Ogle WO, Sapolsky RM. Stress and depression: Possible links to neuron death in the hippocampus. Bipolar Disord. 2002;4:117-128. https:doi.org/10.1034/ j.1399-5618.2002.01144.x.

21. Stubbs DJ, Yamamoto AK, Menon DK. Imaging in sepsis-associated encephalopathy-insights and opportunities. Nat Rev Neurol. 2013;9:551-561. https://doi.org /10.1038/nrneurol.2013.177.

22. Xu XE, Liu L, Wang YC, Wang CT, Zheng Q, Liu QX, et al. Caspase-1 inhibitor exerts brain-protective effects against sepsis-associated encephalopathy and cognitive impairments in a mouse model of sepsis. Brain Behav Immun. 2019;80:859-870. https://doi.org/10.1016/j.bbi.2019.05.038.

23. Fu Q, Wu J, Zhou XY, Ji MH, Mao QH, Li Q, et al. Nlrp3/caspase-1 pathway-induced pyroptosis mediated cognitive deficits in a mouse model of sepsis-associated encephalopathy. Inflammation. 2019;42:306-318. https://doi.org/10.1007/s10753 -018-0894-4.

24. Zhang H, Wei M, Lu X, Sun Q, Wang C, Zhang J, et al. Aluminum trichloride caused hippocampal neural cells death and subsequent depression-like behavior in rats via the activation of il-1beta/jnk signaling pathway. Sci Total Environ. 2020;715:136942. https://doi.org/10.1016/ j.scitotenv.2020.136942. 
25. Zhou RX, Li YY, Qu Y, Huang Q, Sun XM, Mu DZ, et al. Regulation of hippocampal neuronal apoptosis and autophagy in mice with sepsis-associated encephalopathy by immunity-related gtpase m1. CNS Neurosci Ther. 2020;26:177-188. https: //doi.org/10.1111/cns.13229.

26. Bolognese AC, Yang WL, Hansen LW, Denning NL, Nicastro JM, Coppa GF, et al. Inhibition of necroptosis attenuates lung injury and improves survival in neonatal sepsis. Surgery. 2018;S0039-6060 (18) 30096-5. https://doi.org/10.1016/j.surg.2018.02.017.

27. Zhang Q, Wei S, Lu J, Fu W, Chen H, Huang Q, et al. Necrostatin-1 accelerates time to death in a rat model of cecal ligation and puncture and massively increases hepatocyte caspase-3 cleavage. Am J Physiol Gastrointest Liver Physiol. 2019;316:G551-G561. https://doi.org/10.1152/ajpgi.00 175.2018.

28. Chen G, Xiao B, Chen L, Bai B, Zhang Y, Xu Z, et al. Discovery of new md2-targeted antiinflammatory compounds for the treatment of sepsis and acute lung injury. Eur $\mathrm{J}$ Med Chem. 2017;139:726-740. https://doi.org/10.1016/j.ejmech.2017.08.036.

29. Brites D, Fernandes A. Neuroinflammation and depression: Microglia activation, extracellular microvesicles and microrna dysregulation. Front Cell Neurosci. 2015;9:476. https://doi.org/10.33 89/fncel.2015.00476.

30. Ng A, Tam WW, Zhang MW, Ho CS, Husain SF, McIntyre RS, et al. II-1 beta, il-6, tnf- alpha and crp in elderly patients with depression or alzheimer's disease: Systematic review and meta-analysis. Sci Rep. 2018;8:12050. https://doi.org/10.1038/s4159 8-018-30487-6.

31. Ramakrishnan P, Baltimore D. Sam68 is required for both nf-kappab activation and apoptosis signaling by the tnf receptor. Mol Cell. 2011;43:167-179. https://doi.org/10.1016/j.molcel. 2011.05.007.

32. Cho SJ, Choi MH, Nam SY, Kim JY, Kim CS, Pyo S, et al. Sam68 is cleaved by caspases under apoptotic cell death induced by ionizing radiation. J Radiat Res. 2015;56:287-293. https://doi.org/ 10.1093/jrr/rru113.

\section{Figures}


A

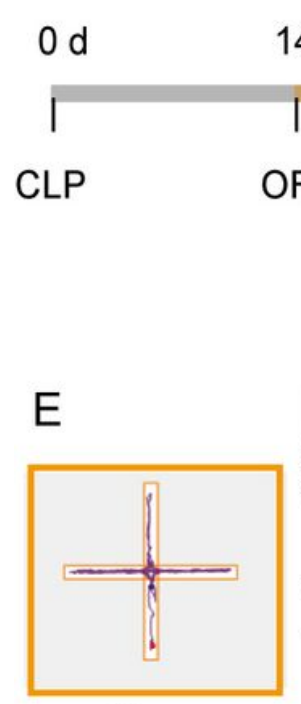

Sham

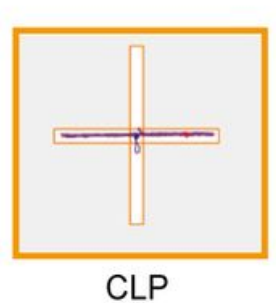

CLP
$14 d \quad 15 d \quad 16 d$

OFT EPM FST

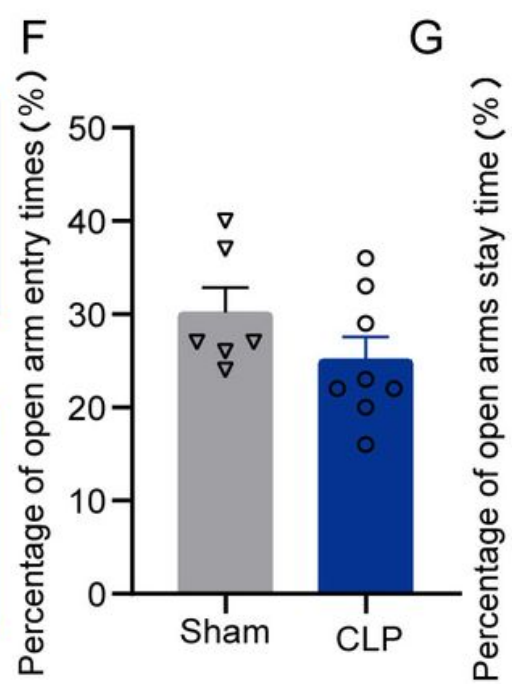

C
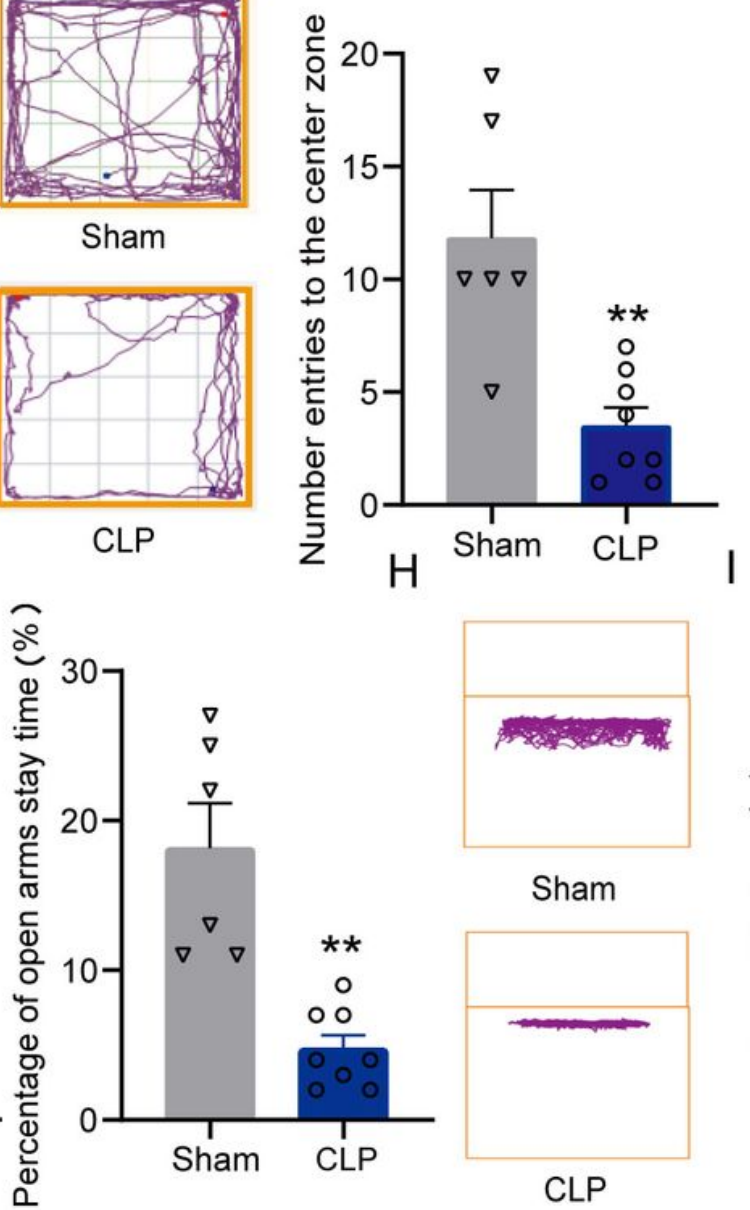

CLP

Sham

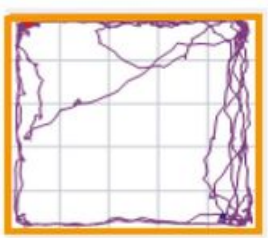

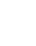

D
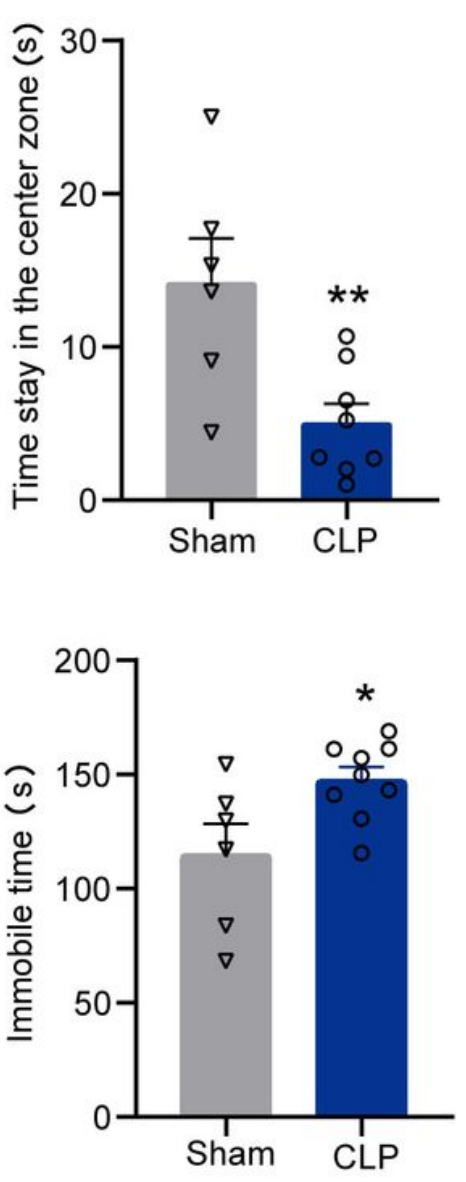

$\mathrm{J}$

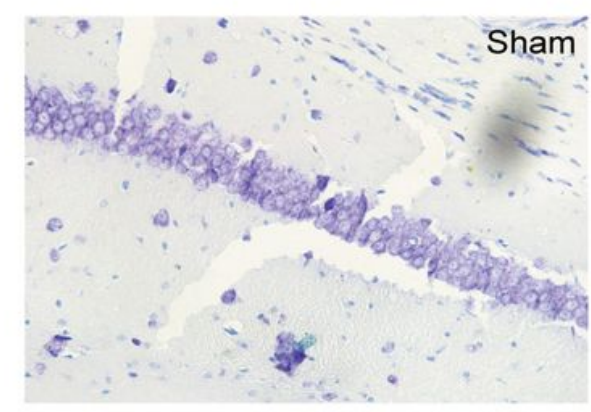


tracking chart of the FST between the sham and CLP groups. I. Total immobility time during 5 min in the FST. ${ }^{*} P<0.05$ vs. the sham group. Data are shown as the mean \pm SAE ( $n$ sham $=6, n$ CLP $=8$ ). J. Nissl staining in the hippocampus of the sham and CLP groups $14 \mathrm{~d}$ after CLP. K. The number of dead neurons in the hippocampus between the sham and CLP groups. ${ }^{\star \star} \mathrm{P}<0.01$ vs. the sham group. Data are shown as the mean $\pm \operatorname{SAE}(n=9)$.

A
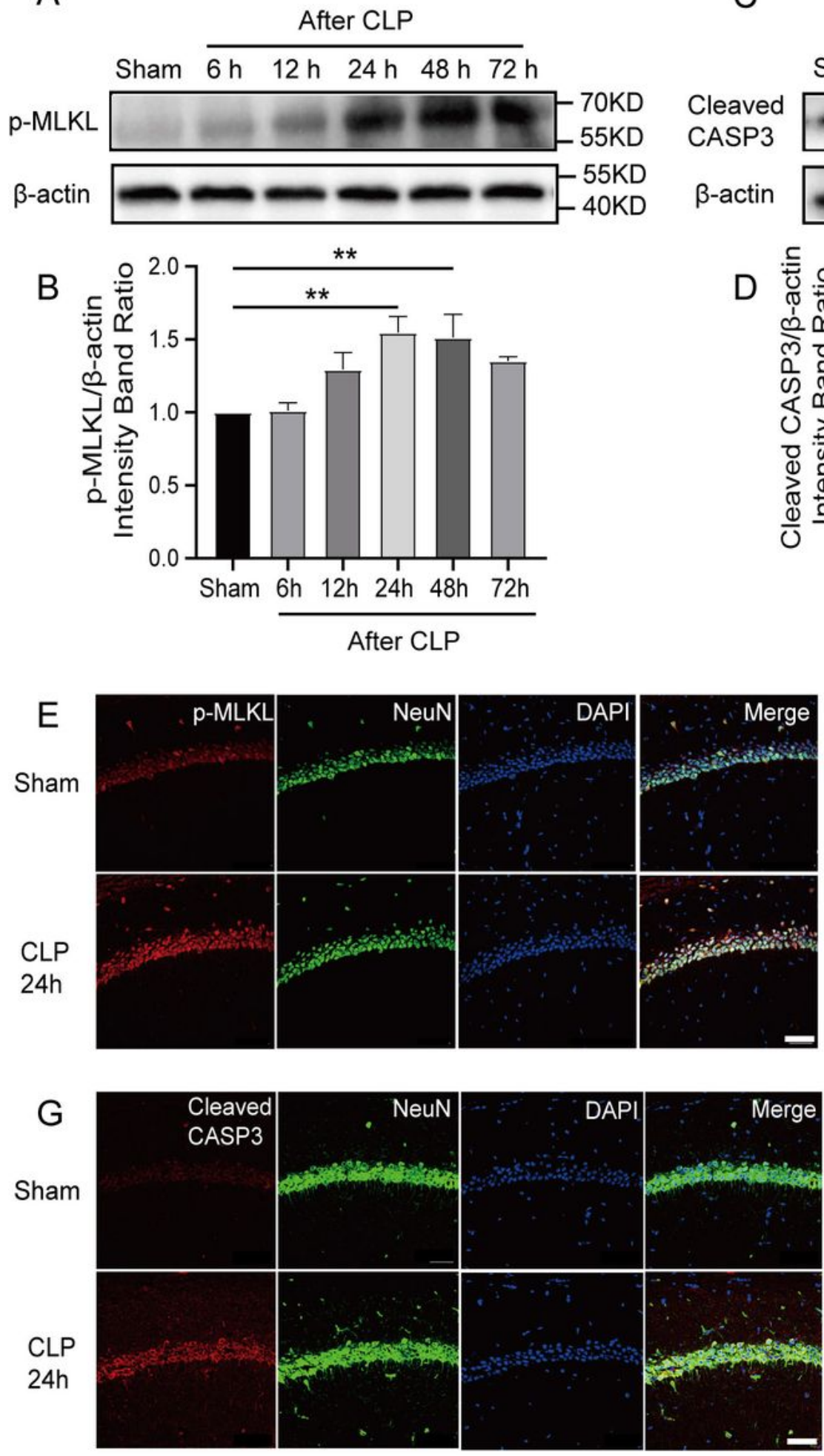

C
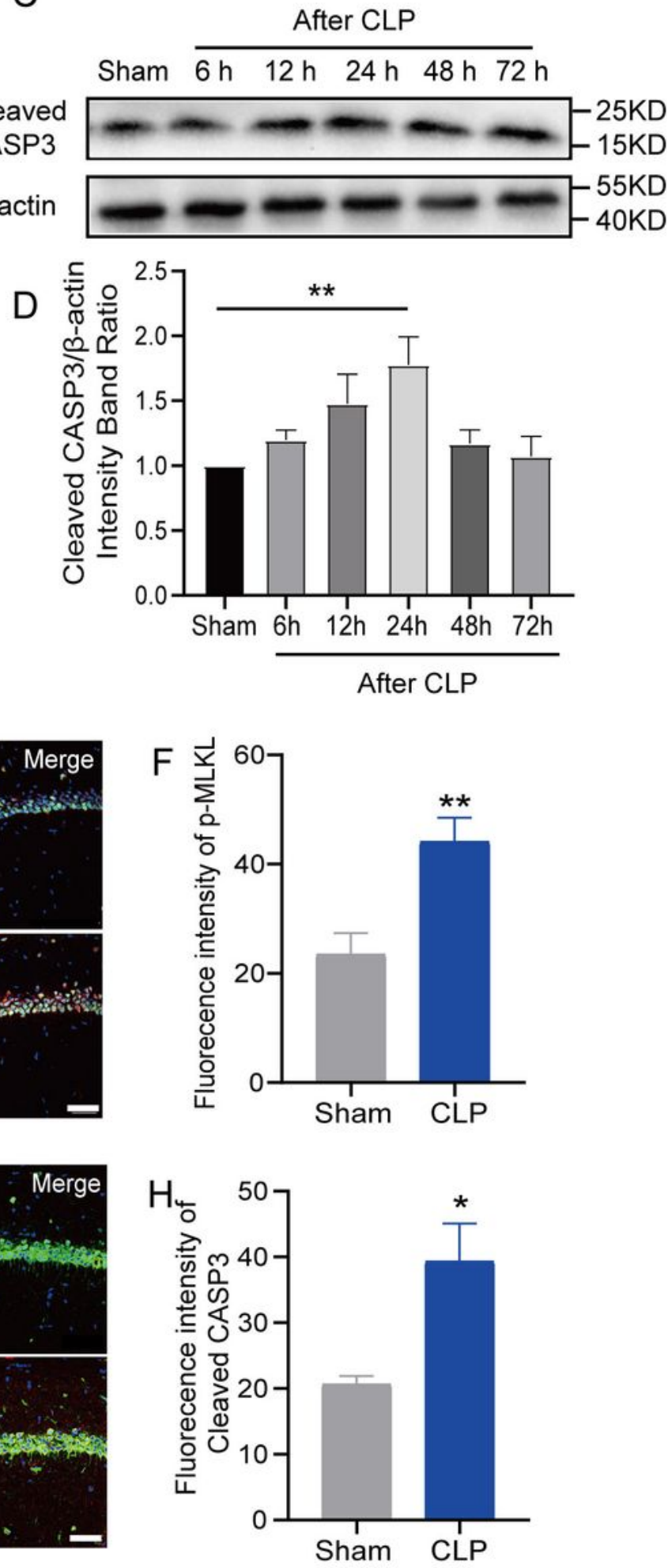

Figure 2 
Apoptosis and necroptosis were increased in the hippocampus of mice with sepsis A. Representative Western blot of p-MLKL. B. Quantitative evaluation of p-MLKL expression after CLP at the time point. ${ }^{*} P<0.01$ vs. the sham group. Data are shown as the mean $\pm S A E(n=9)$. C. Representative Western blot of cleaved caspase-3. D. Quantitative evaluation of cleaved caspase-3 expression after CLP at the time point. ${ }^{*} P<0.01$ vs. the sham group. Data are shown as the mean $\pm S A E(n=9)$. E. IF staining of $p-M L K L 24$ $\mathrm{h}$ after CLP. F. Analysis of the intensity of $\mathrm{p}-\mathrm{MLKL}$ between the sham and CLP groups. ${ }^{\star \star P}<0.01 \mathrm{vs}$. the sham group. Data are shown as the mean $\pm \operatorname{SAE}(n=9)$. G. IF staining of cleaved caspase-3 $24 \mathrm{~h}$ after CLP. $\mathrm{H}$. Analysis of the intensity of cleaved caspase- 3 between the sham and CLP groups. ${ }^{*}<0.05$ vs. the sham group. Data are shown as the mean $\pm \operatorname{SAE}(n=9)$.

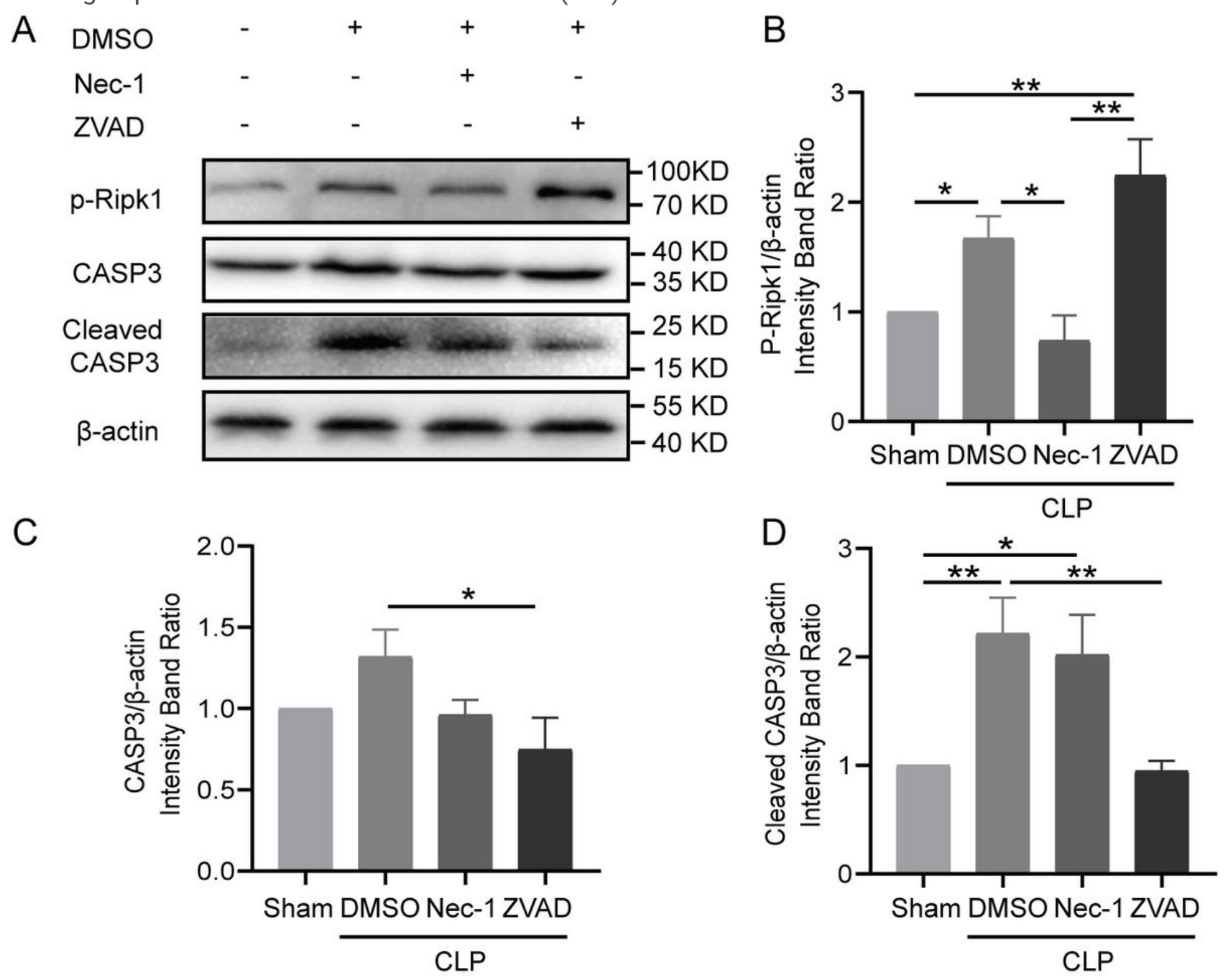

Figure 3

Apoptosis and necroptosis showed Ramsay's rule in the brains of septic mice A. Representative Western blots of p-Ripk1, caspase-3 and cleaved caspase-3. B. Quantitative evaluation of p-Ripk1 expression with different treatments. C. Quantitative evaluation of caspase-3 expression with different treatments. D. 
Quantitative evaluation of cleaved caspase-3 expression with different treatments. ${ }^{*} P<0.05,{ }^{*} \mathrm{P}<0.01$. Data are shown as the mean $\pm \operatorname{SAE}(n=4)$.

A
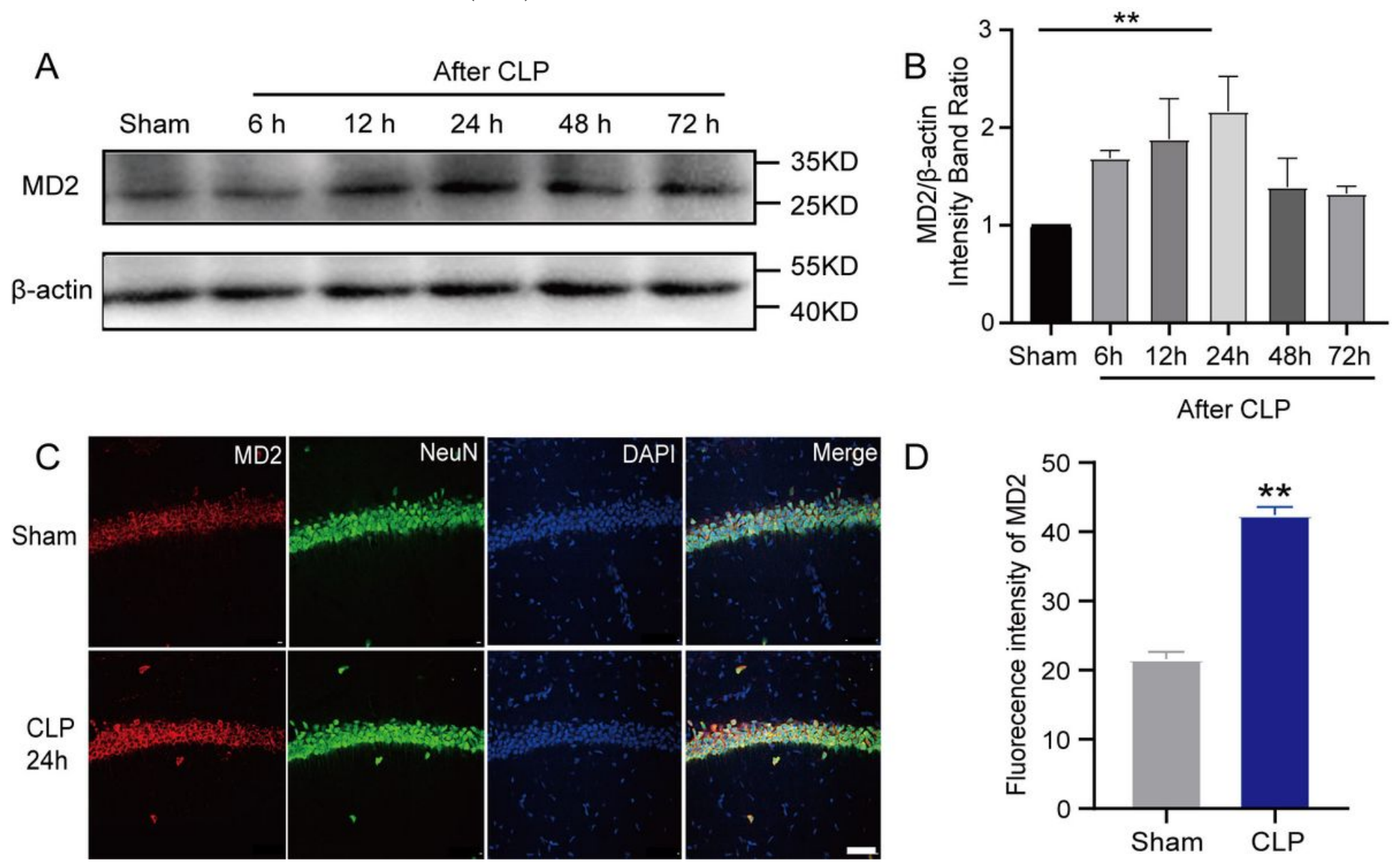

Figure 4

MD2 was increased in hippocampal neurons in septic mice A. Representative Western blot of MD2. B. Quantitative evaluation of MD2 expression after CLP at the time point. ${ }^{*} \mathrm{P}<0.01$ vs. the sham group. Data are shown as the mean $\pm S A E(n=9)$. C. IF staining of MD2 $24 \mathrm{~h}$ after CLP. D. Analysis of the intensity of MD2 between the sham and CLP groups. ${ }^{\star *} \mathrm{P}<0.01$ vs. the sham group. Data are shown as the mean $\pm S A E$ $(n=9)$. 
A

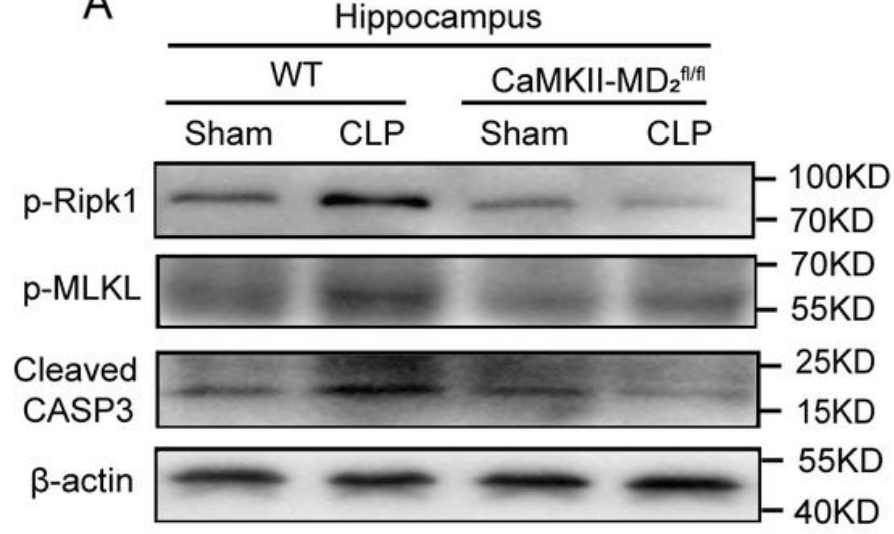

$E$

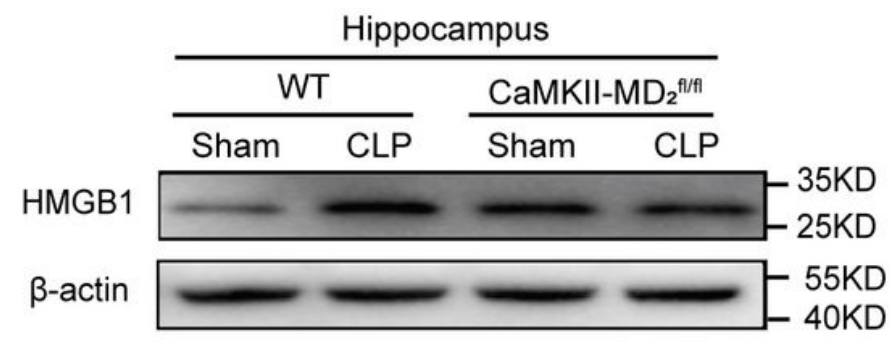

G
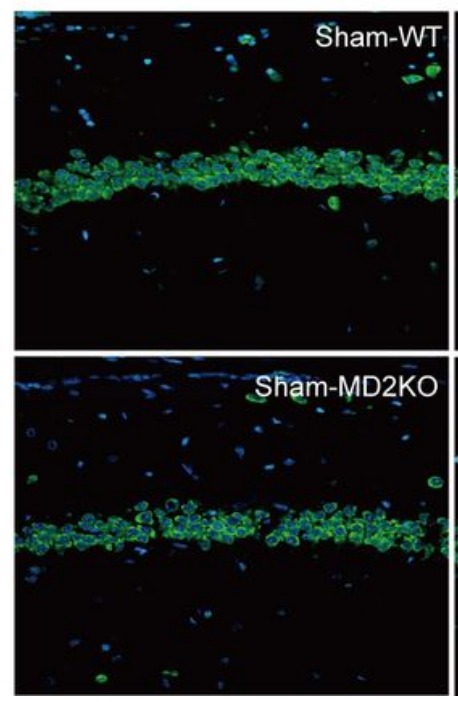

B

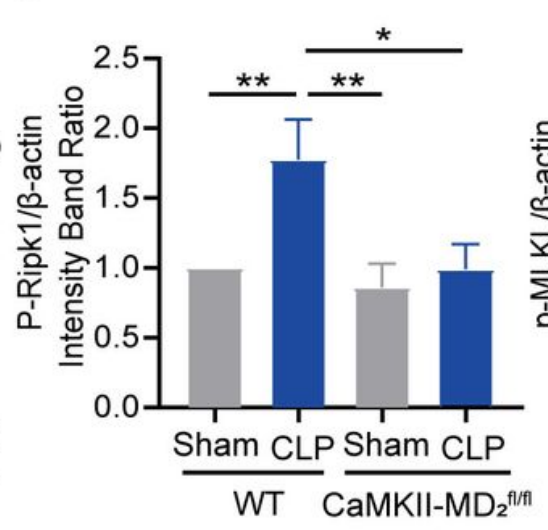

C

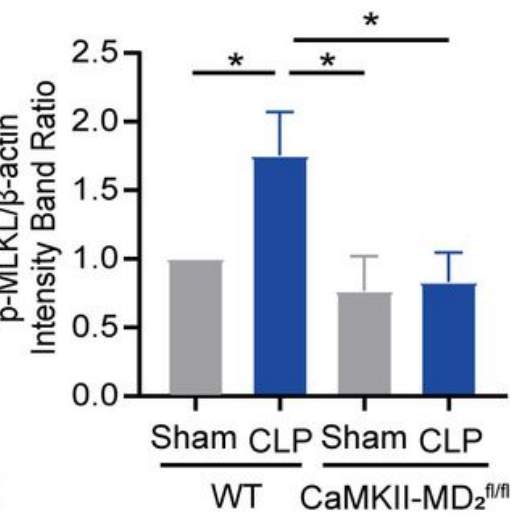

$\mathrm{F}$
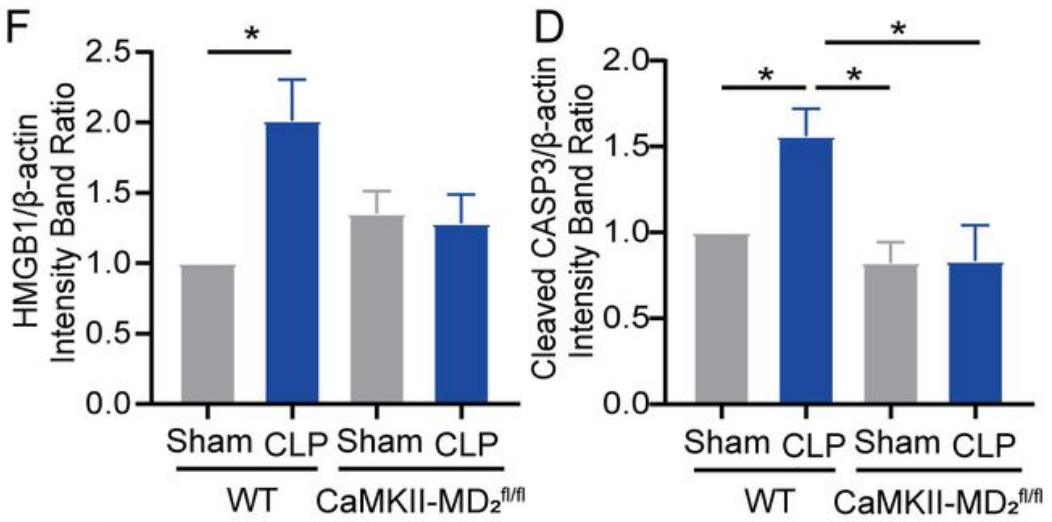

$\mathrm{H}$

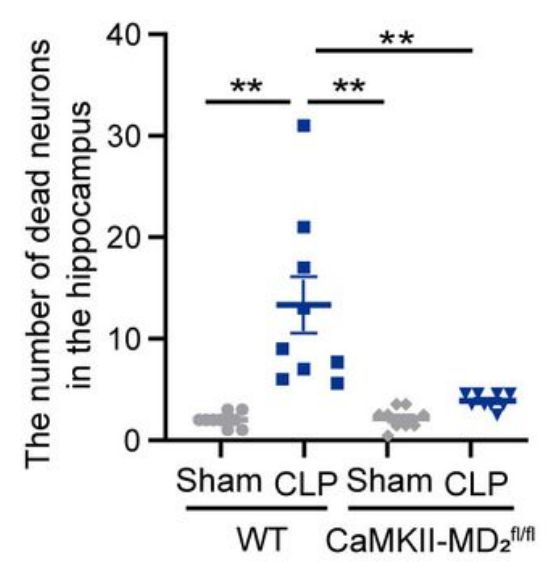

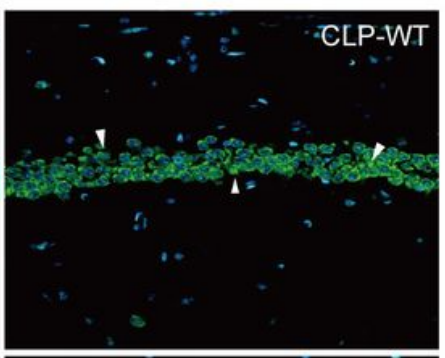

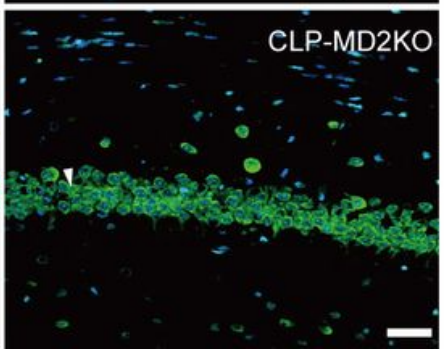

\section{Figure 5}

MD2 synchronously regulated apoptosis and necroptosis in hippocampal neurons, and inhibition of MD2 reduced cell death A. Representative Western blots of $p$-Ripk1, p-MLKL and cleaved caspase-3 in different groups. B. Quantitative evaluation of p-Ripk1 expression. C. Quantitative evaluation of p-MLKL expression. D. Quantitative evaluation of cleaved caspase-3 expression $24 \mathrm{~h}$ after CLP in different groups. ${ }^{*} P<0.05,{ }^{* * P}<0.01$ vs the CLP-WT group. Data are shown as the mean $\pm S A E(n=8)$. E. Representative Western blot of HMGB1 $24 \mathrm{~h}$ after CLP. F. Quantitative evaluation of HMGB1 $24 \mathrm{~h}$ after CLP. ${ }^{*} \mathrm{P}<0.05$. Data are shown as the mean $\pm S A E(n=8)$. G. NeuroTraceTM Nissl staining in the hippocampus at 14 days of 
CLP. H. The number of dead neurons in the hippocampus among different groups. **P<0.01. Data are shown as the mean \pm SAE $(n=9)$.

A Sham-WT

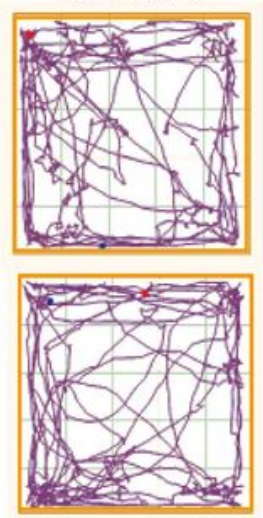

ShamCAMKII-MD ${ }_{2}^{\text {fIIf }}$

D

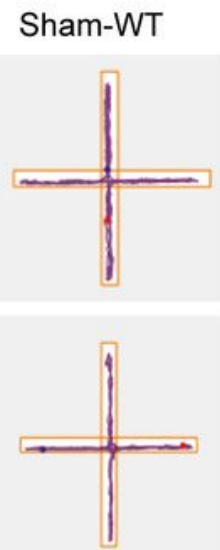

ShamCAMKII-MD ${ }^{\text {fl/f }}$
CLP-WT
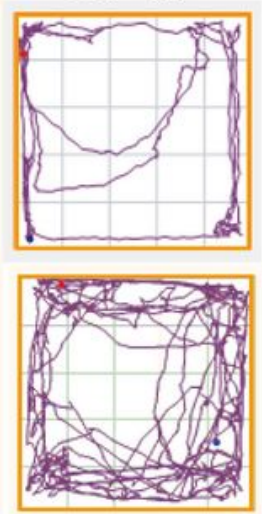

CLP-

CAMKII-MD ${ }_{2}^{\text {fl/ }}$
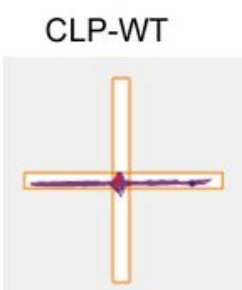

G

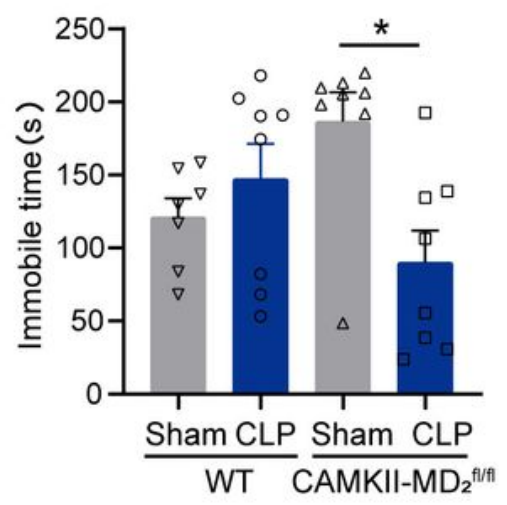

CLP-

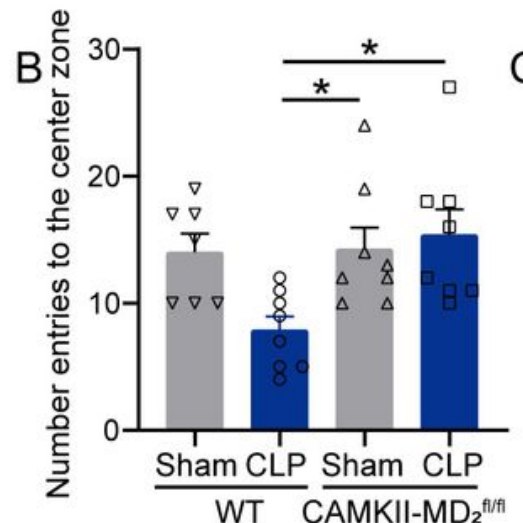

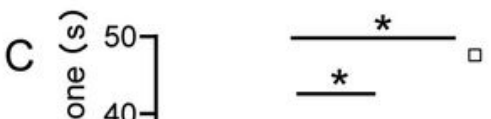
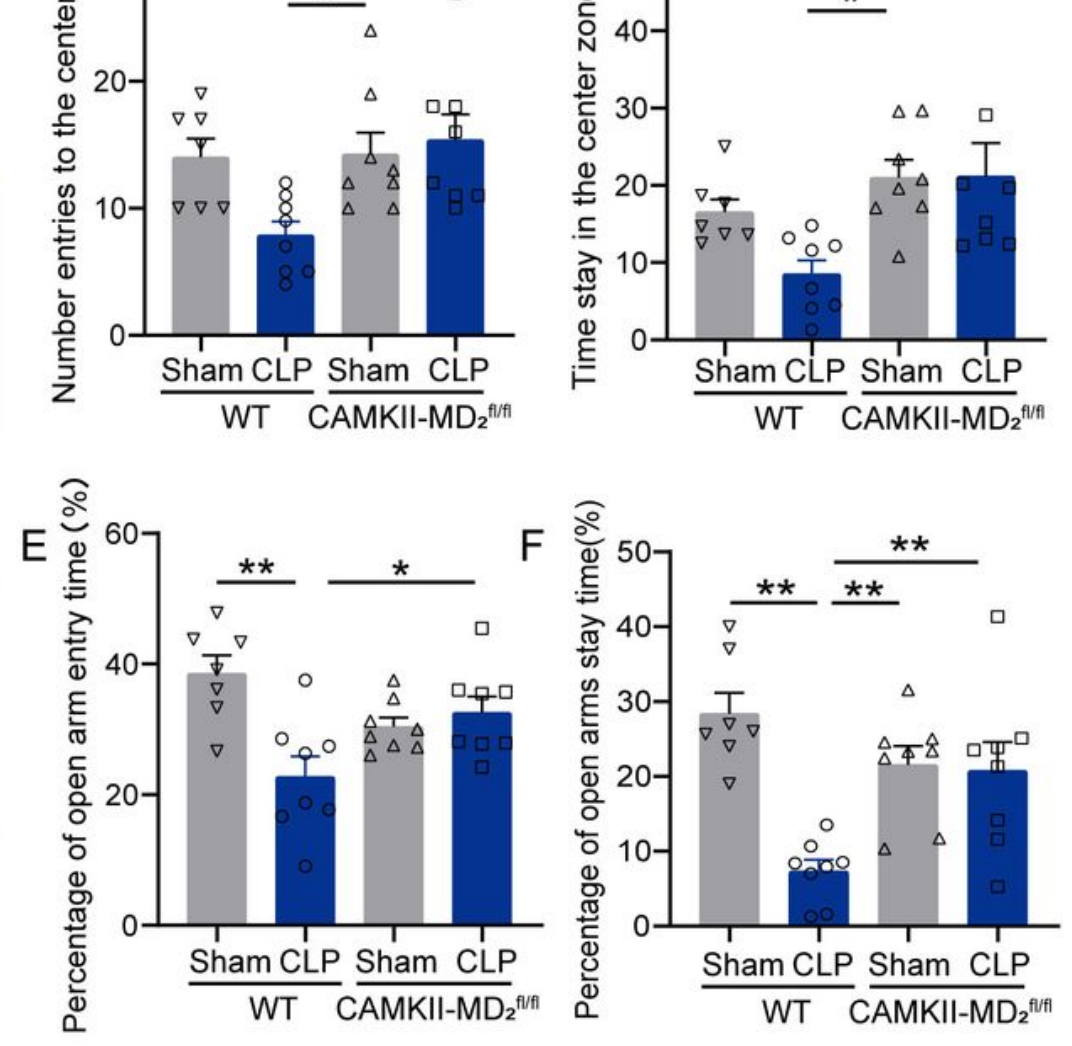

CAMKII-MD ${ }_{2}^{\text {fl/f }}$ SAMKII-MD ${ }_{2}^{\text {fulf }}$
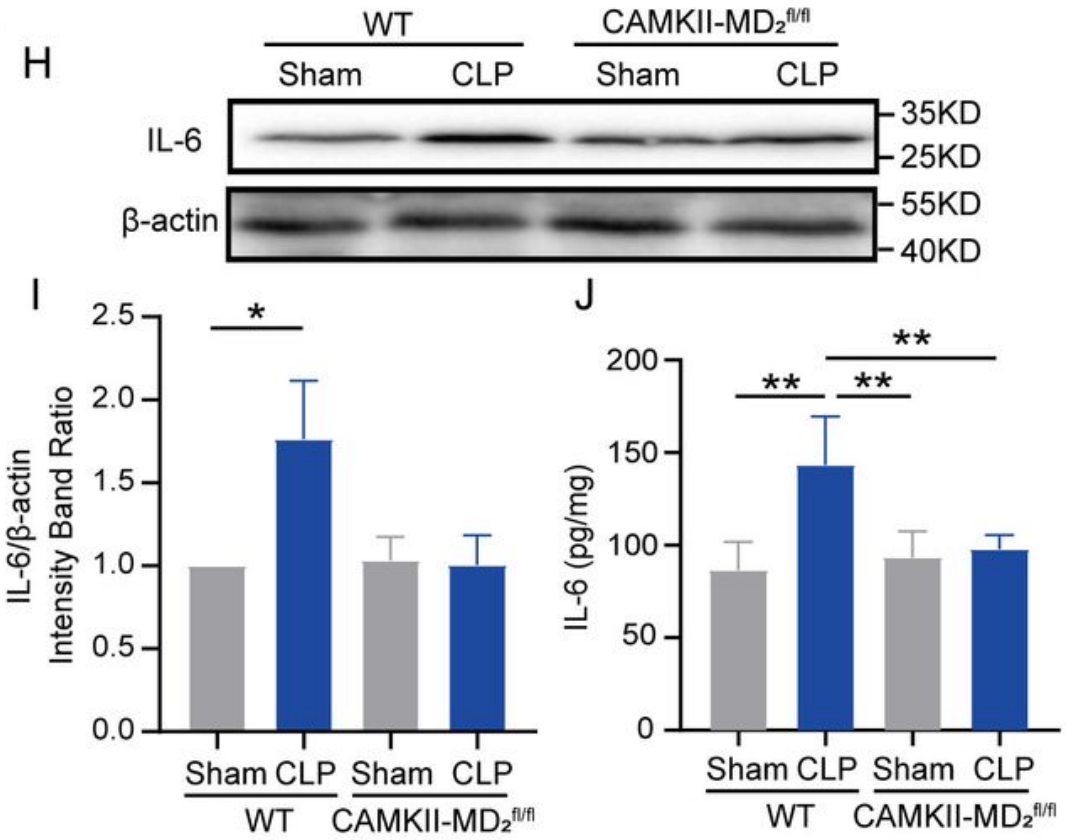

Figure 6

Depression-like behaviors were reduced in the CaMKII-MD2fl/fl mice at $14 \mathrm{~d}$ after CLP A. Typical tracking chart of the OPF between the CaMKII-MD2fl/fl mice and the WT mice. B. The differences between the WT and CaMKII-MD2fl/fl groups in number of entries to the center zone. C. The differences in time spent in 
the center zone in the OFT. ${ }^{*} P<0.05$. Data are shown as the mean \pm SAE ( $n$ sham-WT=7, others $\left.n=8\right)$. $D$. Typical tracking chart of the EPM between the CaMKII-MD2fl/fl mice and the WT mice. E. The percentage of entries to the open arms. F. The percentage of time spent in the open arms. Data are shown as the mean $\pm S A E$ ( $n$ sham-WT $=7$, others $n=8$ ). G. Total immobility time over 5 min in the FST among different groups. ${ }^{*} P<0.05$. H. Representative Western blots of IL-6 $24 \mathrm{~h}$ after CLP. I. Quantitative evaluation of IL- 6 in the hippocampus. ${ }^{*} P<0.05$. Data are shown as the mean $\pm S A E(n=8)$. J. ELISA analysis of IL- 6 in the hippocampus among different groups. ${ }^{*} P<0.01$. Data are shown as the mean $\pm S A E(n=8)$.

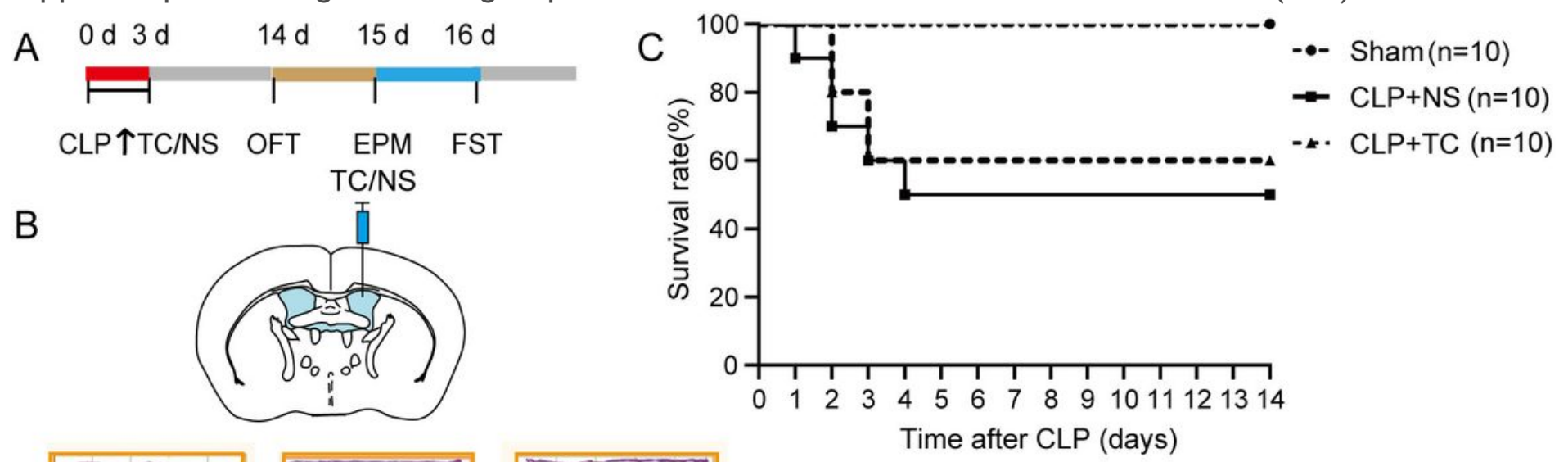

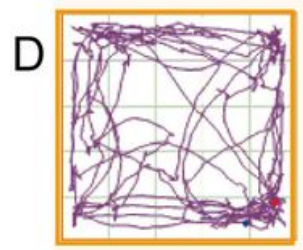

Sham

G

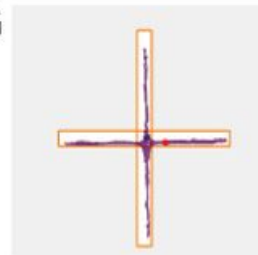

Sham

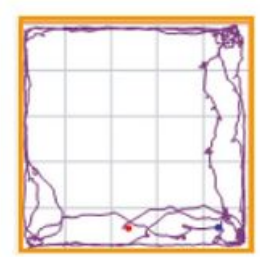

$\mathrm{CLP}+\mathrm{NS}$

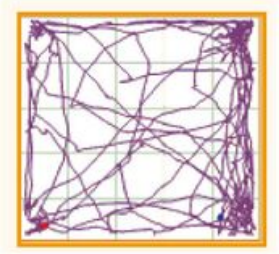

$\mathrm{CLP}+\mathrm{TC}$

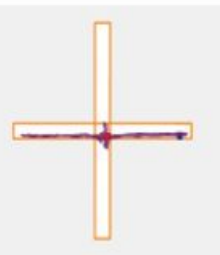

$\mathrm{CLP}+\mathrm{NS}$

$\mathrm{H}$

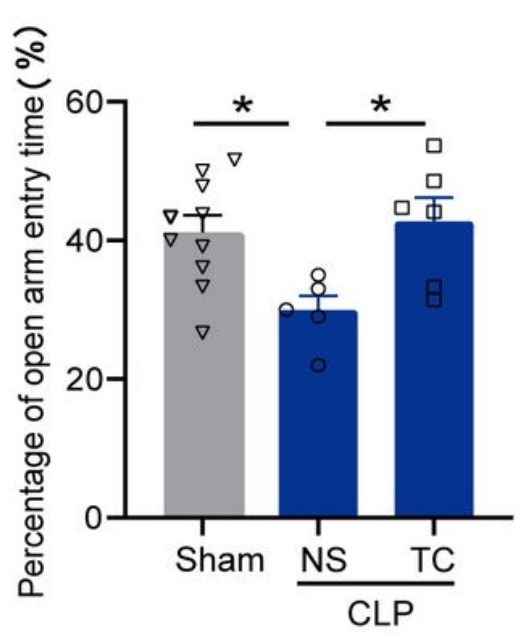

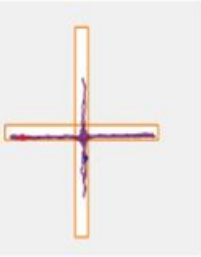

CLP+TC
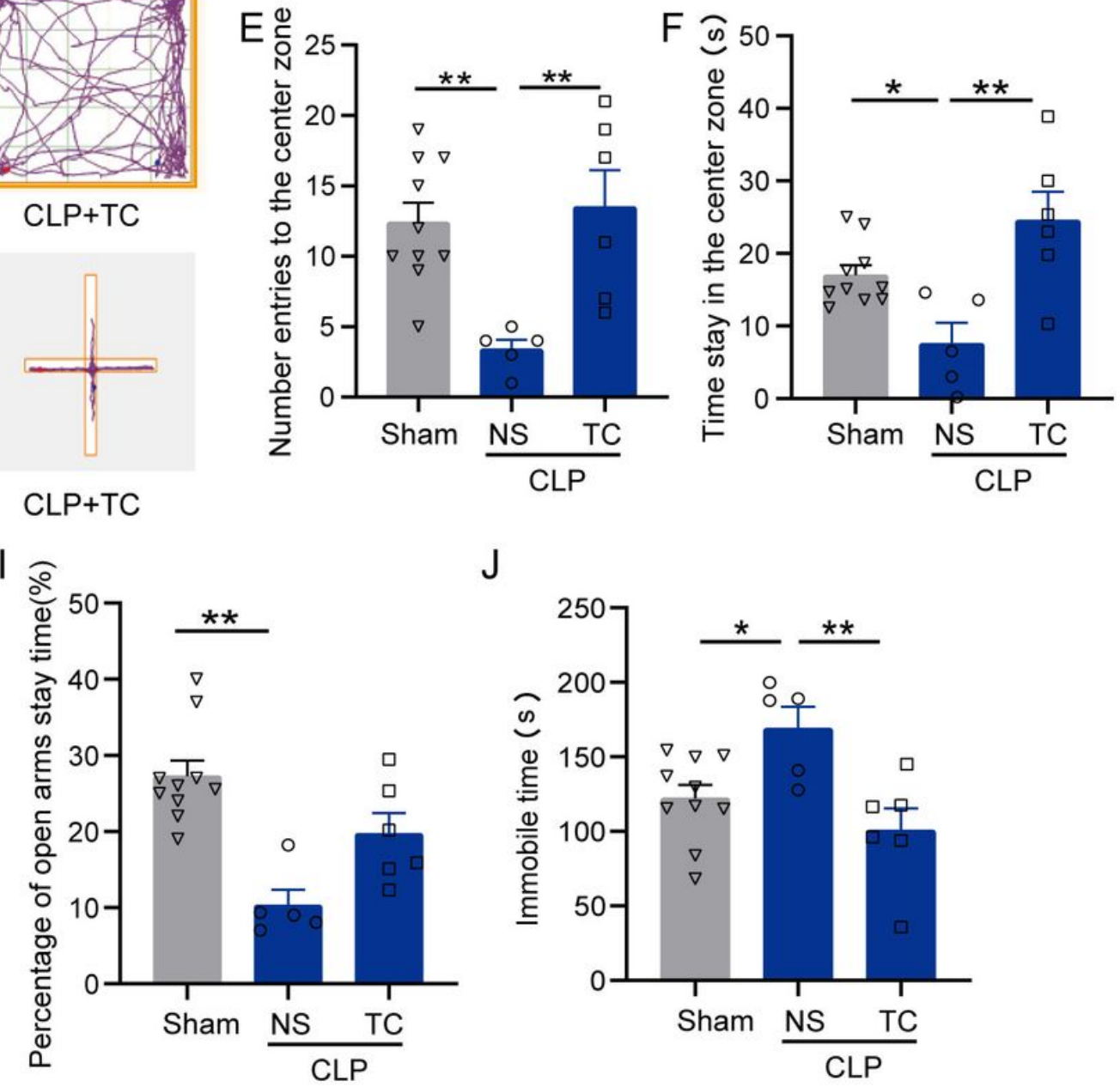

Figure 7 
TAT-CIRP alleviated depression-like behaviors in mice with sepsis A. An outline of the experimental procedure for TC injection and behavioral tests of mice with sepsis. B. Schematic configuration of icv injection. C. Survival curve for TC icv injection. Five of 10 mice in the CLP+NS group survived by day $14 ; 6$ of 10 mice in the CLP+TC group survived. D. The representative tracking chart of the OFT. E. The effects of different treatments on number of entries to the center zone. F. The time spent in the center zone in the OFT. ${ }^{*} P<0.05,{ }^{\star *} P<0.01$. Data are shown as the mean $\pm S A E$ ( $n$ sham $=9, n$ CLP+NS $=6, n$ CLP+TC=6). $G$. The representative tracking chart of the EPM. H. In the EPM, percentage of entries to the open arms. I. The percentage of time spent in the open arms. ${ }^{*} P<0.05,{ }^{* *} P<0.01$. Data are shown as the mean \pm SAE $(n$ sham $=9, \mathrm{n} C L P+N S=6, \mathrm{nCLP}+\mathrm{TC}=6$ ). $\mathrm{J}$. Total immobility time over $5 \mathrm{~min}$ in the FST among different groups. Data are shown as the mean $\pm S A E$ ( $n$ sham $=9, n C L P+N S=6, n C L P+T C=6$ ).
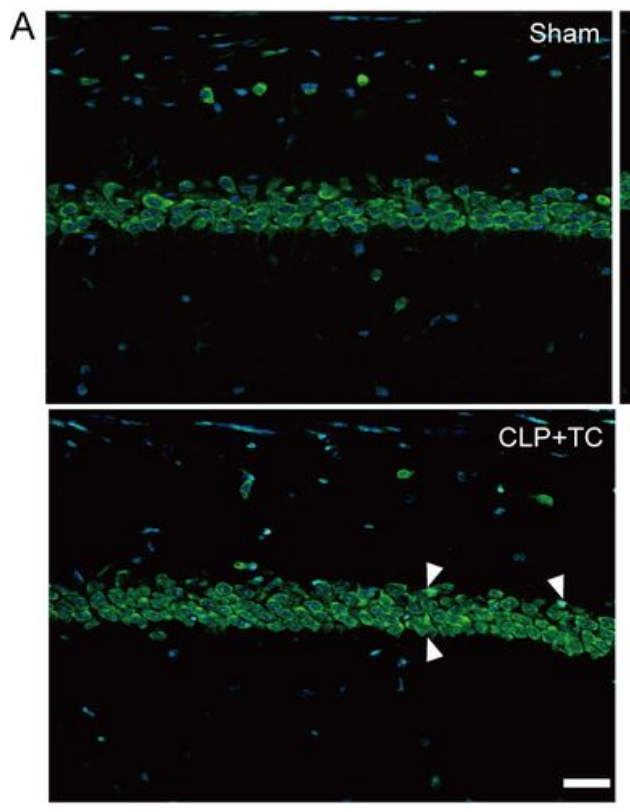
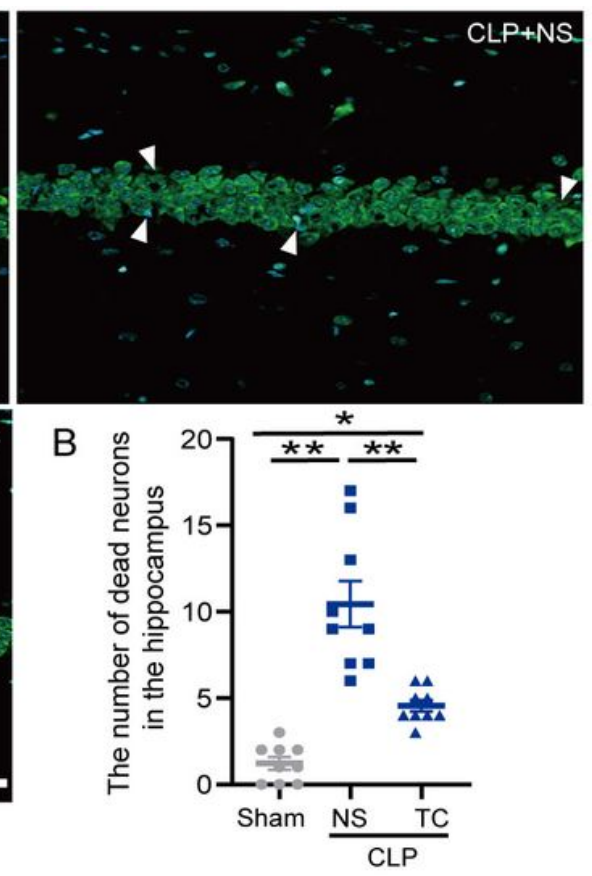

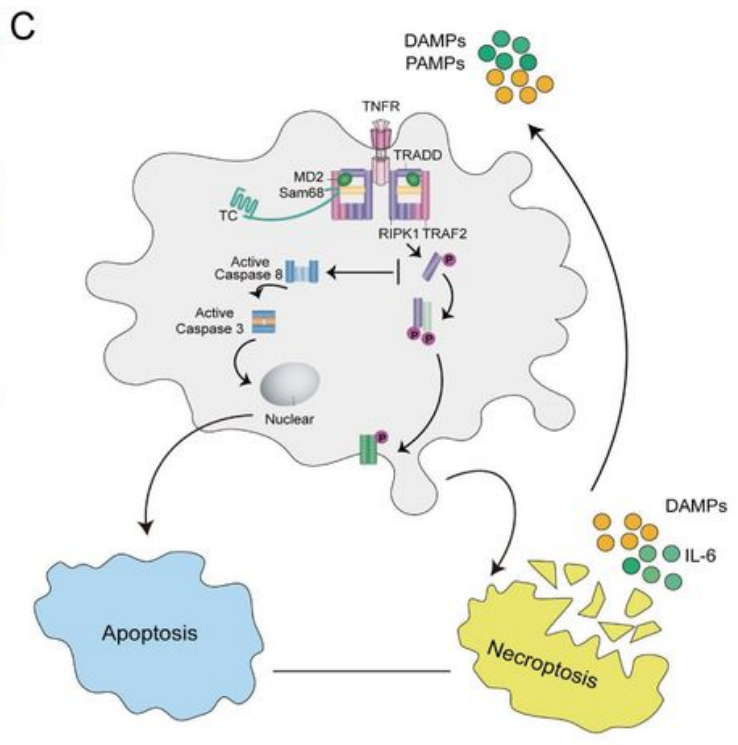

\section{Figure 8}

Neuronal loss in the hippocampus was reduced in the TAT-CIRP-treated septic mice A. NeuroTraceTM Nissl staining in the hippocampus at 14 days after CLP. B. The number of dead neurons in the hippocampus among different groups. ${ }^{*}<0.05$, ${ }^{\star *} P<0.01$. Data are shown as the mean $\pm S A E(n=9)$. C. The graphical abstract. In sepsis, MD2 in neurons is activated and combines with Sam68 to trigger apoptosis, and the necroptosis pathway results in neuronal death; neuronal death releases many DAMPs and further exacerbates cell death. TC inhibits cell death by disturbing the binding of MD2 and Sam68.

\section{Supplementary Files}

This is a list of supplementary files associated with this preprint. Click to download.

- SupplementaryFigure1.tif

- SupplementaryFigure2.tif 
- SupplementaryFigure3.tif

- SupplementaryFigure4.tif

- SupplementaryFigure5.tif

- SupplementaryFigure6.tif

- SupplementaryFigure7.tif

- SupplementaryTable1.tif

- SupplementaryTable2.tif 